\title{
A GMM-BASED STAIR QUALITY MODEL FOR HUMAN PERCEIVED JPEG IMAGES
}

\author{
Sudeng Hu, Haiqiang Wang and C.-C. Jay Kuo \\ Media Communications Laboratory, University of Southern California
}

\begin{abstract}
Based on the notion of just noticeable differences (JND), a stair quality function (SQF) was recently proposed to model human perception on JPEG images. Furthermore, a k-means clustering algorithm was adopted to aggregate JND data collected from multiple subjects to generate a single SQF. In this work, we propose a new method to derive the SQF using the Gaussian Mixture Model (GMM). The newly derived SQF can be interpreted as a way to characterize the mean viewer experience. Furthermore, it has a lower information criterion (BIC) value than the previous one, indicating that it offers a better model. A specific example is given to demonstrate the advantages of the new approach.
\end{abstract}

Index Terms - Stair quality function (SQF), just noticeable difference (JND), Gaussian mixture model (GMM), JPEG

\section{INTRODUCTION}

The traditional quality metric for coded image/video, such as the peak signal-to-noise ratio (PSNR), is a continuous function of the coding bit rate [1, 2, 3, 4]. Several newly proposed quality metrics such as SSIM [5] and perceptually weighted PSNR [6] still preserve this property. However, these continuous quality models contradict to our subjective visual experience since human can only differentiate a small number of quality levels.

Based on the notion of just noticeable difference (JND), it was shown in [7] that human-perceived quality of JPEG images is a stair function of the quality factor (QF). It is a monotonically increasing piecewise-constant function characterized by a couple of jumps. The stair quality function (SQF) is discontinuous, and its jumps can be interpreted as the JND points between two adjacent quality levels. For a given image coded by JPEG with multiple QFs, the number of discrete quality levels and the location of JND points vary among test subjects. Since they are random variables, it is important to develop a methodology to integrate the data collected from multiple test subjects. A simple k-means clustering algorithm was proposed in [7] to process collected JND data to generate the aggregate SQF, which is called the K-SQF here.

In this work, we treat JND points from a subject as samples, and use the Gaussian Mixture Model (GMM) to fit the sample distribution. This approach leads to another aggregate SQF, called the G-SQF. The G-SQF can be interpreted as the mean viewer experience in differentiating compressed image quality under a wide range of coding bit rates. The shape parameters of the G-SQF such as the number of discrete quality levels and the location and height of JND points are determined automatically by this modeling procedure. As compared to the ad hoc k-means clustering algorithm used in deriving the K-SQF, the G-SQF is rooted in solid theoretical foundation. We will show that the G-SQF has a lower information criterion (BIC) value than the K-SQF, indicating that it is a better model. Furthermore, a specific example will be given to demonstrate the advantages of G-SQF over K-SQF.
This rest of this paper is organized as follows. The data collection procedure for JND-based subjective JPEG quality assessment is reviewed in Section 2. A GMM-based processing technique is proposed to handle collected JND data in Section 3. The performance comparison of G-SQF and K-SQF is conducted in Section 4. Finally, concluding remarks are given in Section 5.

\section{JND-BASED SUBJECTIVE JPEG QUALITY ASSESSMENT}

The process of building a large-scale human-centric quality dataset for JPEG-coded images, called MCL-JCI, is described below. MCLJCI contains 50 source (or uncompressed) images of resolution 1920x1080. Each source image is coded by the JPEG encoder [8] 100 times with the quality factor (QF) set from $1,2,3 \ldots$ to 100 . Thus, the whole MCL-JCI dataset consists of 5,050 images in total. The quality of coded images with respect to each source image is evaluated by 20 subjects. They were seated in a controlled environment. The viewing distance was 2 meters ( 1.6 times the picture height) from the center of the monitor to the seat. The image pair was displayed on a 65 " TV with native resolution of $3840 \times 2160$. A subject compared two images displayed side by side and determined whether these two images are noticeably different.

The following bisection search procedure was adopted to offer a more robust and efficient pairwise comparison result.

- Initialization. We begin with comparing images of the best and the worst quality. The best quality is obtained by setting $\mathrm{QF}=100$ while the worst quality is set to the $\mathrm{QF}$ value that gives the lowest acceptable through subjective test. Before the subjective test, a small number of volunteers were asked to find the lowest acceptable QF parameter.

- Iteration. Compare two images whose QF is located at two ends of the interval of interest and see whether they have noticeable difference or not. If no, no further search is needed for this interval since it does not contain a JND point. If yes, we partition the interval into two halves of equal length, and repeat the same comparison procedure iteratively until one of the two termination criteria is reached.

- Termination. There are two termination cases. First, the process is terminated when the interval length reaches the minimum value with the QF difference equal to one. Second, one observes noticeable difference at a certain level and cannot observe any noticeable difference at the next level.

The above subjective test produces raw JND data samples for each image, where one subject contributes a set of JND samples. The histograms of JND points for two exemplary images are shown in Fig. 1 where (a) and (b) are obtained from source image No. 6 and No. 26, respectively. They are too complicated to be used as is. It is essential to process them and build an aggregate SQF for each 


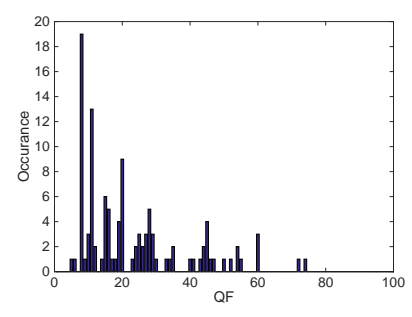

(a)

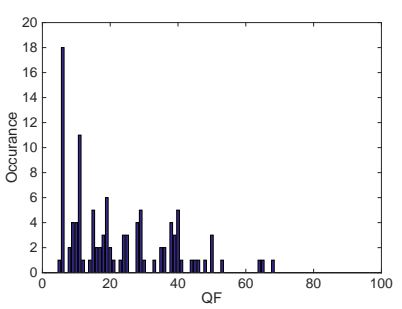

(b)
Fig. 1: The histograms of JND points for source image (a) No. 06 and (b) No. 26.

individual image. Ideally, the aggregate SQF can be used to characterize the mean experience of subjects in the test. The derivation of an accurate SQF will facilitate the use of the machine learning technique in predicting the SQF for images not in the dataset.

The k-means clustering algorithm was proposed in [7] to process collected JND samples for an aggregate SQF, where $k$ is determined by the rounded mean of distinguishable quality levels. However, the k-means clustering approach is a heuristic one. It is difficult to give the resulting SQF any statistical meaning. To address this shortcoming, we propose a new method to process collected JND samples based on the GMM. Mathematically, it is easy to see that the resulting SQF offers the mean viewer experience among all subjects participating in the test.

\section{GMM-BASED JND DATA PROCESSING}

\subsection{Group Partitioning of JND Points}

Images coded in the range of high QFs have good perceptual quality and their distortion can hardly be perceived. As a result, there are only few JND points falling in this range as compare to those in the low QF range. This phenomenon is obvious in the exemplary histograms given in Fig. 1 Because perceptual difference in high quality images is so small that it can be easily neglected when compared with low quality images in statistical analysis. Furthermore, compressed images with high QF values are more important in practice since people are interested in high quality images in most applications. They are much more frequently used than those compressed with low QF values. For the above-mentioned reasons, the high QF JND points should not be merged with the low QF JND points to form components in one single GMM. By following a similar argument, one can argue that JND points in the low QF range should not be merged with those in the middle QF range. As a result, we classify JND samples into three main groups according to their locations: high QF, middle QF and low QF groups. This is achieved by a partitioning scheme described below.

First, we order JND points according to their QF values (from the largest to the smallest) and identify the JND points lying at the top $10 \%$ and $50 \%$ locations. Then, we examine the heights of these two JND points against the JND histogram curve. There are three scenarios.

1. If a JND point happens to be a local minimum (or zero), we select it as the boundary point between two groups.

2. If a JND point is a local maximum, we search two local minima (or zero) along its left and right directions and select the smaller one as the updated boundary point.

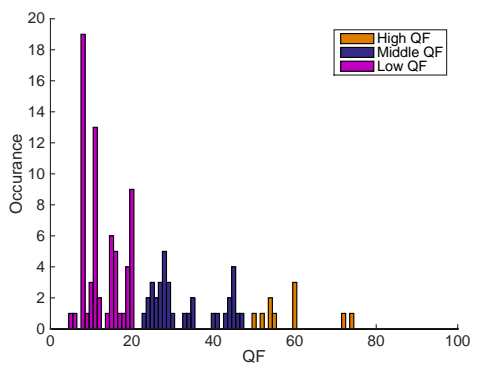

Fig. 2: Partitioning of JND points into high, middle and low QF groups, where each group will be modeled by a GMM independently.

3. If the JND point is neither a local maximum nor a local minimum, we search along the descending direction for the local minimum (or zero) and select it as the updated boundary point.

We split the height of a boundary point (i.e. its number count) equally into two halves - one goes to the left and the other goes to the right. By following the above steps, we obtain the high, middle and low QF groups. An example is given in Fig. 2 Then, we will use three GMMs to model their JND distributions independently.

\subsection{JND Histogram Modeling with GMM}

The number of distinguishable quality levels and their JND positions depend on both image content and test subjects. Even for the same image and the same QF group, it is still difficult to group JND points since different subjects may have different numbers of JND points. To proceed with statistical analysis, we need an underlying model for the JND distribution. Here, it is assumed that the JND distribution is in form of GMM with $N$ components. Mathematically, it can be expressed as

$$
f(x)=\sum_{i=1}^{N} \alpha_{i} \cdot \frac{1}{\sqrt{2 \pi} \sigma_{i}} \exp \left(-\frac{\left(x-q_{i}\right)^{2}}{2 \sigma_{i}^{2}}\right),
$$

where each component is a normal distribution with mean $q_{i}$ and variance $\sigma_{i}^{2}$, and $a_{i}$ is the mixture weight satisfying the constraint $\sum_{i=1}^{N} \alpha_{i}=1$.

To determine the set of parameters of GMM in Eq. (1); namely,

$$
\Theta=\left\{\alpha_{i}, q_{i}, \sigma_{i}\right\}, \quad i=1, \cdots, N,
$$

we adopt the Expectation Maximization (EM) algorithm [9]. It is well known that the EM algorithm is an iterative algorithm that updates these parameters in each iteration until the process converges or reaches the preset maximum iteration number. The EM algorithm is sensitive to the initial values of these parameters. In the proposed method, we compute the histogram of JND samples in the target QF region, and select the location of $N$ largest bins as initial value for the mean of $N$ components (i.e., $q_{i}, i=1, \cdots, N$ ). The initial variance of all components is set to 1 .

Furthermore, we need to specify the component number, $N$, of the GMM. If $N$ is too small, it is difficult to fit the JND samples well. If $N$ is too large, it may result in overfit. Here, we perform an exhaustive search for the optimal component number $N^{*}$. That is, we begin with $N=1$, and increase its value by one every time until $N$ reaches the pre-set maximum component number of each group. 


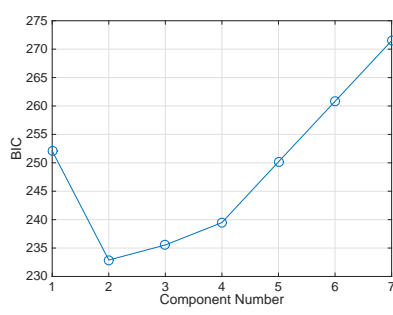

(a)

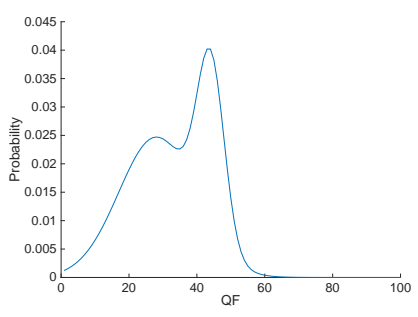

(b)

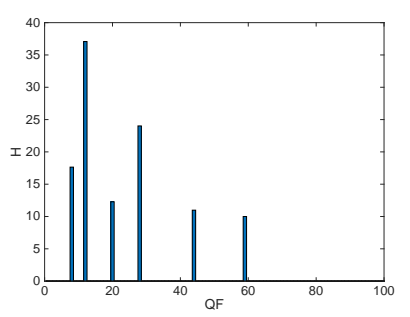

(a)

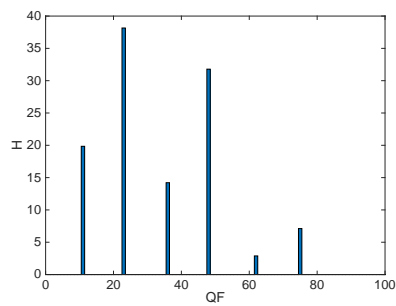

(c)

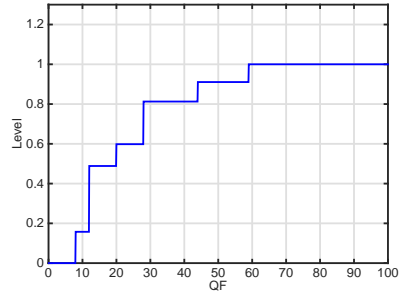

(b)

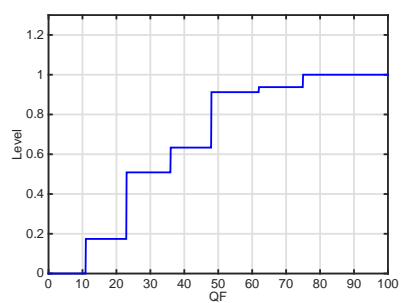

(d) under control.

We use the Bayesian information criterion (BIC) [10] to determine the best GMM. A lower BIC value indicates better performance. Mathematically, the BIC is defined as

$$
\mathrm{BIC}=-2 \cdot \ln (\hat{L})+k \cdot \ln (n),
$$

where $\hat{L}$ is the maximized value of the likelihood function of the model, $\ln$ is the natural $\log , k$ is the number of free parameters in the model, and $n$ is the number of samples. In the current case,

$$
\hat{L}=p(x \mid \Theta),
$$

where $x$ denotes all samples and $\Theta$ is the set of GMM parameters. Both terms of BIC in Eq. (2) are positive. A better fit will drive the first term lower and a smaller $k$ will drive the second term lower for fixed $n$. Thus, the BIC value helps strike a balance between data fitting performance and model complexity.

To give an example, for the middle QF group in Fig. 2, we show the BIC value as a function of the GMM component number, $N=1, \cdots, 7$, in Fig. 3 (a). The BIC decreases as the component number increases from $N=1$ to 2 . The BIC reaches the minimum value at $N=2$. Afterwards, the BIC increases as $N$ increases. The probability density function of the optimal GMM with $N=2$ is shown in Fig. 3 (b), where we see two Gaussian components clearly.

\subsection{From GMM to SQF}

Once a GMM is built for each QF group (or region), the remaining task is to build the corresponding SQF. We first discuss the SQF for a single QF group. Let the normal function $N\left(x \mid q_{i}, \sigma_{i}\right)$ be the $i$ th Gaussian function in the GMM in the corresponding region. We associate the location of the $i$ th jump in the SQF with $q_{i}$ while its height is set to be proportional to the area under the weighted normal function $\alpha_{i} N\left(x \mid q_{i}, \sigma_{i}\right)$.

Next, we examine the SQF for all three QF regions combined together. The JND for the whole range can be obtained by combining the three JND sets. Mathematically, the JND function can be written as

$$
J N D(x)=\sum_{j=1}^{3} \sum_{i=1}^{N} H_{i j} \delta\left(x-q_{i j}\right),
$$

Fig. 4: (a) The simplifed JND histogram of source image No. 06, (b) the SQF of source image No. 06, (c) the simplifed JND histogram of source image No. 26, (d) The SQF of source image No. 26.

where $\delta(\cdot)$ is the Dirac delta function and $H_{i j}$ is the percepture quality change degree at the $i$ th JND position in the $j$ th group (i.e. low, middle and high QF groups). The SQF is the normalized cumulative sum of JND function. Mathematically, it can be expressed as

$$
S Q F(x)=\frac{1}{\sum_{j=1}^{3} \sum_{i=1}^{N} H_{i j}} \int_{0}^{x} J N D(t) d(t),
$$

which is a monotonically increasing piecewise constant stair function.

To give an example, after the processing of the two raw JND histograms shown in Figs. 1 (a) and (b) obtained from 20 subjects, we can aggregate them into two simplified JND histograms as shown in Fig. 4 (a) and (c) while their corresponding SQFs are shown in Figs. 4 (b) and (d), respectively. These two SQFs offer the mean viewer experience towards these two images.

\section{EXPERIMENTAL RESULTS}

In this section, we compare the performance of the method proposed in [7], called the K-method, and the proposed JND data processing method, called the G-method. The BIC in Eq. (2) offers a relative estimate of information loss in using a statistical model to model sample data. It is used to compare the performance of two models quantitatively. To calculate BIC of the K-method, a statistical model is needed to model JND points. We use a Gaussian model for each JND in the K-method. The JND location from the K-method is set to the mean of the Gaussian model and the model variance is set to the sample variance of that cluster. The BIC is calculated for all 50 images in the data set, and the results are shown in Fig. 5 We see the BIC of G-method is always lower than that of the K-method, which means the G-method offers a better model.

Without loss of generality, we choose source image No. 13 in Fig. 5 as an example to shed light on the BIC values of the two methods. The BIC value consists of two terms as presented in Eq. (2). 


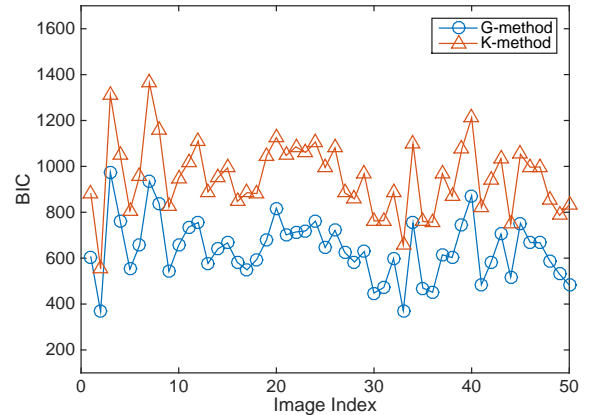

Fig. 5: Comparison of the BIC values of two models with respect to 50 source images, where the K-method and the G-method denote the k-means clustering method used in [7] and the proposed GMMbased method, respectively.

One is the goodness of fit to the sample data, which is determined by the negative model log-likelihood term. Another term is the penalty of model complexity that is related to the number of model parameters. The BIC value and its two contributing terms are listed in Table 1 for two models on image No. 13. The BIC value of the K-method is 888.84 , which is larger than that of the G-method. By examining their individual contributing terms, we see that the difference in their model complexity penalty terms are relatively minor as compared to that of the model negative log-likelihood term. It shows that the Kmethod does not offer a good model for JND points.

Table 1: Comparison of the model negative log-likelihood term, the model complexity term and the BIC values of the K-method and the G-method for Image No. 13.

\begin{tabular}{c|ccc}
\hline & $-2 \ln (P(x \mid \Theta))$ & Complexity & BIC \\
\hline K-method & 824.65 & 64.19 & 888.84 \\
G-method & 485.37 & 91.70 & 577.07 \\
\hline
\end{tabular}

We compare the performance of two methods for Image No. 13 side by side in Fig. 6 The first, second and third rows of the figure display the input JND histograms before modeling, the output JND histograms after modeling, and the final SQFs. The difference in histograms in Fig. 6 (a) and (b) is due to a weighting scheme used in the K-method. In that method, the JND point from each individual was first normalized by the total observed JND numbers. (For example, if a person observes $N$ JND points, each JND point of him/her is weighted by $1 / N$.) This scheme penalizes observed JND data in the high QF region since people who observe JND points in the high $\mathrm{QF}$ region tend to have a larger total JND number. In contrast, we do not perform any weighting on the collected JND data. The JND histogram in Fig. 6(b) is obtained with raw user data.

The output JND histograms obtained by the two methods are shown Fig. 6(c) and (d), respectively. In the K-method, the location of the output JND point is determined as the median of JND points within that cluster. These points are marked by circles in Fig. 6(a). This may lead to inaccurate result when JND points are not clustered correctly. For example, the first four JND points (from the left) in Fig. 6 (c) are very close to each other while the last JND points is very far apart from the others. In our proposed method, the output JND locations are set to the means of all Gaussian components. They are more stable and set apart with more uniform spacing.

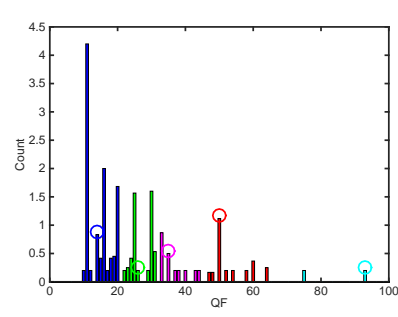

(a)

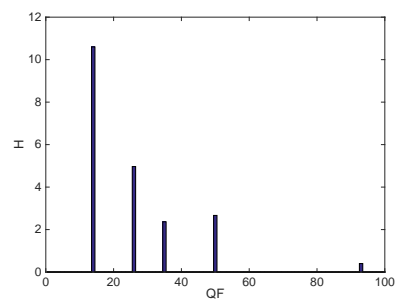

(c)

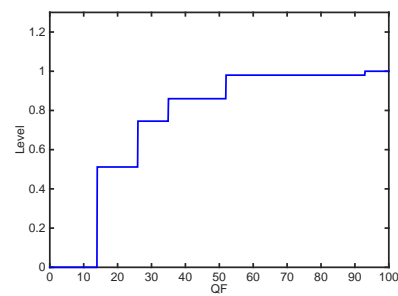

(e)

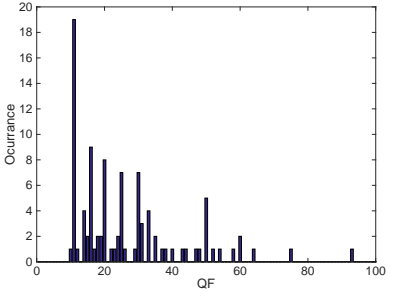

(b)

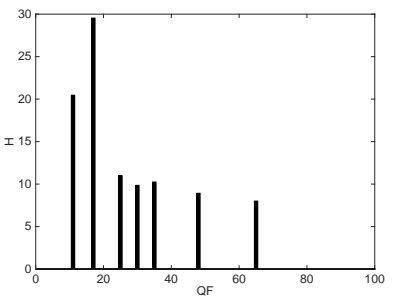

(d)

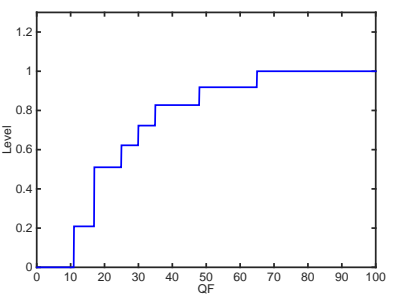

(f)
Fig. 6: (a) The input weighted JND histogram used in the K-method, (b) the input raw JND histogram used in the G-method, (c) the output JND histogram processed by the K-method, (d) the output JND histogram processed by the G-method, (e) the K-SQF, and (f) the G-SQF.

It is worthwhile to point out that the total JND number for a given image in the $\mathrm{K}$-method is a pre-set number. This ad hoc choice has a negative impact on its output JND histogram as shown in Fig. 6 (c). It has 5 peaks only, which is less than the G-method by two. In the proposed G-method, the JND number is determined by optimizing the model with the lowest BIC value. Finally, Fig. 6 (e) and (f) show the K-SQF and the G-SQF, respectively. The G-SQF offers more quality levels, which is actually more reasonable by reexamining the full set of JPEG coded images for Image No. 13.

\section{CONCLUSION AND FUTURE WORK}

In this work, we proposed the use of the GMM to model the raw JND data collected by the subjective test, and derived the G-SQF. The new method always provides a model that has a smaller BIC value than that proposed in [7], indicating that it is a better model. The G-SQF for all 50 source images will be made available to the public soon. It will provide a training dataset of human perceptual experience on JPEG images. We will develop a machine learning technique to predict viewer experience on JPEG images that are not in the training dataset. 


\section{REFERENCES}

[1] Damon M Chandler and Sheila S Hemami, "VSNR: A waveletbased visual signal-to-noise ratio for natural images," IEEE Transactions on Image Processing, , vol. 16, no. 9, pp. 22842298, 2007.

[2] Yung-Kai Lai and C-C Jay Kuo, "A Haar wavelet approach to compressed image quality measurement," Journal of Visual Communication and Image Representation, vol. 11, no. 1, pp. 17-40, 2000.

[3] M. Masry, S.S. Hemami, and Y. Sermadevi, "A scalable wavelet-based video distortion metric and applications," IEEE Transactions on Circuits and Systems for Video Technology, vol. 16, no. 2, pp. 260-273, Feb 2006.

[4] K. Seshadrinathan and A.C. Bovik, "Motion tuned spatiotemporal quality assessment of natural videos," IEEE Transactions on Image Processing, , vol. 19, no. 2, pp. 335-350, Feb 2010.

[5] Zhou Wang, Alan Conrad Bovik, Hamid Rahim Sheikh, and Eero P Simoncelli, "Image quality assessment: from error visibility to structural similarity," IEEE Transactions on Image Processing, vol. 13, no. 4, pp. 600-612, 2004.

[6] Sudeng Hu, Lina Jin, Hanli Wang, Yun Zhang, Sam Kwong, and C.-C. Jay Kuo, "Compressed image quality metric based on perceptually weighted distortion," IEEE Transactions on Image Processing, to appear.

[7] Joe Yuchieh Lin, Lina Jin, Sudeng Hu, Ioannis Katsavounidis, Zhi Li, Anne Aaron, and C.-C. Jay Kuo, "Experimental design and analysis of JND test on coded image/video," Proc. SPIE 9599, Applications of Digital Image Processing XXXVIII, pp. 95990Z-95990Z-11, September 2015; doi:10.1117/12.2188389.

[8] Independent JPEG Group, "JPEG image compression software," http: / /www.ijg.org. Accessed: 2015-03-20.

[9] Arthur P Dempster, Nan M Laird, and Donald B Rubin, "Maximum likelihood from incomplete data via the em algorithm," Journal of the royal statistical society. Series B (methodological), pp. 1-38, 1977.

[10] David F Findley, "Counterexamples to parsimony and bic," Annals of the Institute of Statistical Mathematics, vol. 43, no. 3, pp. 505-514, 1991. 